\title{
Synthesis of Lick Spectral Indices With Overshooting and No-Overshooting Model Atmospheres
}

\author{
R.K. Gulati and M. Chavez \\ Instituto Nacional de Astrofisica Optica y Electronica, Apdos. Postales \\ 51 y 216, CP 72000 Puebla, Mexico
}

\begin{abstract}
Using Lester's version of the Kurucz codes, we have computed a set of models with and without "approximate overshooting". Based on the new models, we have synthesized the spectra in the wavelength range 4800-5400 $\AA$ in order to investigate the role of overshooting and no-overshooting models on the Lick indices. Preliminary results show that the differences between the indices are significant in the case of $\mathrm{H}_{\beta}$ and $\mathrm{Mg}_{b}$. We need to investigate further the importance of this convection parameter, by comparing model spectra with observed spectra.
\end{abstract}

\section{Introduction}

Spectral indices serve as a probe for understanding the basic physical properties of individual stars and the stellar contents of the local group of galaxies. Among the several spectral indices, Lick indices have been widely used to interpret the stellar contents of elliptical galaxies using stellar population synthesis models. Ever since the indices were introduced by Faber (1973), they have been refined, modified and supplemented from time to time by Faber and her co-workers and the most recent list of the indices is given in Table 2 of Trager et al. (1998).

In evolutionary population synthesis models, Worthey (1992) has used the indices in the form of fitting functions, derived from the behavior of the empirical indices with respect to atmospheric parameters, together with evolutionary models to understand the history and chemistry of the stellar contents of old populations. However, the empirical library of spectra from which the indices are measured suffer due to incompleteness in sections of the parameter space and due to the non-uniqueness of the parameters associated with each observed spectrum. These drawbacks in empirical libraries have motivated the use of synthetic libraries of spectra based on model atmospheres. The extrapolation of an empirical library to a synthetic library can be safe only after a rigorous test of synthetic spectra with the existing observational data.

Certain applications of the indices require accuracy in their determination, for example Worthey (1994) pointed out that in modeling integrated populations of $10 \mathrm{Gyr}$ and metallicity around $[\mathrm{Fe} / \mathrm{H}]=0.3$, typical errors of $0.20 \AA$ in the Lick indices, $\mathrm{Fe} 5270$ and $\mathrm{H}_{\beta}$, will translate into errors on metallicity $[\mathrm{Fe} / \mathrm{H}]$ and age of the order of 0.18 dex and 6 Gyr respectively. As the Kurucz models are improved upon with the availability of new line data and improved input physics, like other indices we expect improvements in the Lick indices. 
We divide the efforts on modeling of the Lick indices in context of model atmospheres into two groups: one based on the MARCS code (Gustaffson et al. 1975) and other using several ATLAS versions of the Kurucz's codes. Models based on the MARCS code have been used to synthesize $\mathrm{Mg}$ features in the wavelength region 4900-5400 $\AA$ (Barbuy et al. 1992) and more recently the full set of Lick indices (Tripicco \& Bell 1995). On the other hand, Kurucz models have been used by Mould (1978), Gulati et al. (1991, 1993), Chavez et al. (1995, 1996) to study the behavior of Lick indices in terms of atmospheric parameters. Most of our work has concentrated on the $\mathrm{Mg}$ and Fe indices. In our previous papers we have also investigated the effects of other less fundamental parameters such as computation resolution and microturbulent velocity on spectroscopic indices.

In a recent paper, Castelli et al. (1997) have demonstrated that the models based on the ATLAS9 code, without considering the convection prescription called "approximate overshooting", better reproduce observational properties than those of overshooting model atmospheres. In this paper we look into the role of overshooting and no-overshooting models in the synthetic Lick indices. We revisit the indices in light of a new set of models computed with the updated version of the ATLAS9 code.

\section{Synthesis of Model Spectra}

Synthesis of model spectra involves the model atmospheres and line data. The reliability of the spectra depends upon how reliable are the models and line data used as an input. In this section we briefly describe the models that we are using in synthesis of model spectra.

\subsection{Computing Model Atmospheres}

Kurucz's models (e.g. 1979, 1992, 1993) have been used over the years for determining physical properties of stars from photometric, spectrophotometric and spectroscopic data since he has strived to improve the models to match with observations. The other reason is that his models together with a set of codes used to compute them are available on the CDROMs so they can be used to produce models at desired atmospheric parameters.

In our first attempt to synthesize one of the Lick indices, Gulati et al. (1991) computed models using the ATLAS8 version of the Kurucz code, with opacity distribution functions without molecular opacity. A comparison of the synthetic $\mathrm{Mg}_{2}$ indices with those measured from the observed spectra showed that though the trends of the indices with respect to temperature could be reproduced, the models were not adequate enough to reproduce the observed indices. Later on, we investigated the consistency of the synthetic indices with observed ones based on the Kurucz (1992) models which included a contribution due to atomic and molecular opacity. We used these models to synthesize the spectra in the wavelength range, 4850-5400 $\AA$, including $\mathrm{Mg}$ and Fe features. Indices based on these models started showing improvement with respect to observations, in the sense that the calibrated synthetic $\mathrm{Mg}_{2}$ indices were closer to those calibrated from the empirical library. Yet there were discrepancies in the other indices. 

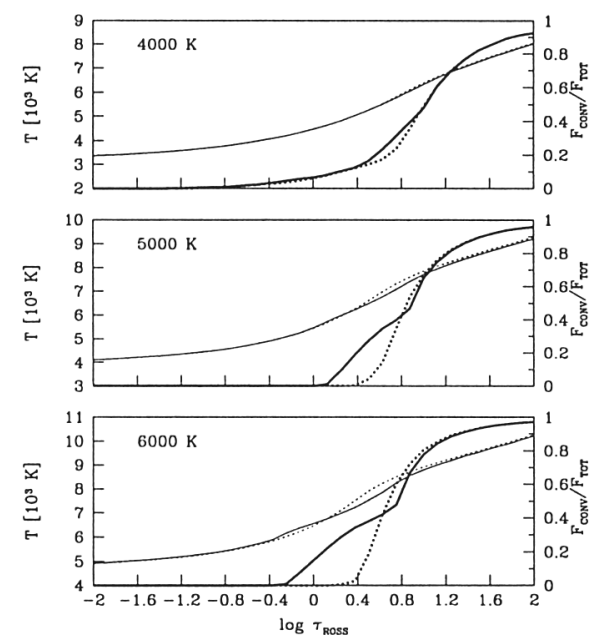

Figure 1. T- $\log \tau_{\text {ROSS }}$ relation (left scale, light lines) and fraction of convective flux as a function of optical depth (right scale, dark lines) for three solar metallicity models of $\log g=4.0$. Solid and dotted lines correspond to models with and without overshooting respectively

Further major improvements and extensions were incorporated in the $\mathrm{Ku}$ rucz codes and models released on CDROM series. New models in CDROM13 included more optical depth points and eliminated the sharp decrease in temperature near the surface. Models, as well as low resolution fluxes, considered revised Fe continuous opacities and a detailed treatment of scattering in the source function. An important extension to this theoretical database was the inclusion of finer steps for super-solar metallicity models. Chavez et al. (1995) have used these models to compute a new grid of synthetic spectra with the same computational parameters as earlier works and have measured the $\mathrm{Mg}_{2}$ indices. Remarkable differences were found, particularly for high metallicity and low gravity models. These new set of $\mathrm{Mg}_{2}$ indices and others reported in Chavez et al. (1996) are shown to be in better agreement with the observations, but still they are unable to show one to one correspondence with the observed indices particularly at low temperatures.

More recently Castelli et al. (1997) have looked deeply into the convective models and analyzed the role of several convection parameters, including overshooting, on Strömgren indices. They concluded that though the overshooting models reproduce observational properties for the sun, yet they are not able to reproduce photometric properties for other stars; and model parameters determined using non-overshooting models are more consistent, irrespective of the methods.

Kurucz models have also been used by others to calibrate broad and narrow band photometric systems to atmospheric parameters. For example, Kunzli et al. (1997) have used them to calibrate the Geneva photometric systems in terms 

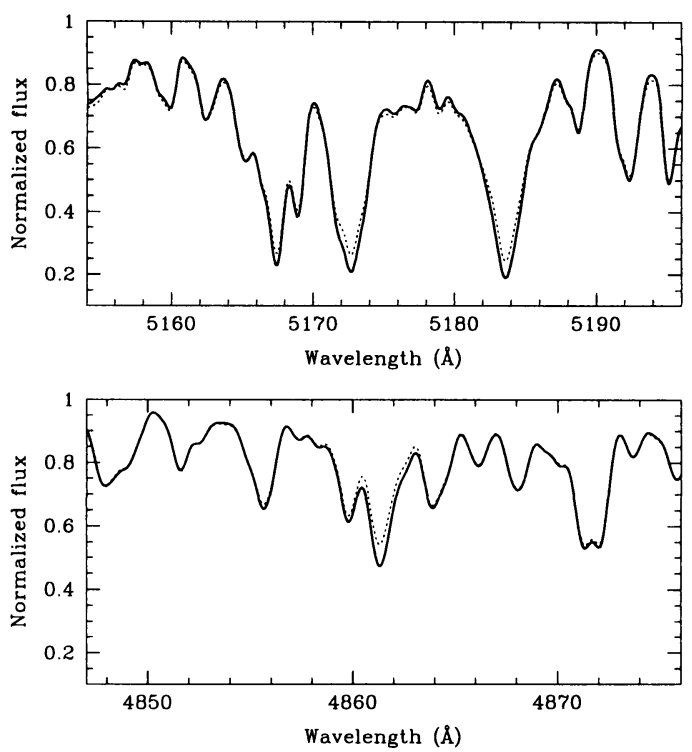

Figure 2. Synthetic spectra in central bands of the $\mathrm{Mg}_{b}$ and $\mathrm{H}_{\beta}$ indices for a stellar model of $5000 \mathrm{~K}, \log g=4.0$ and solar composition. Solid and dotted lines correspond to fluxes with and without approximate overshooting respectively

of effective temperature, surface gravity and metallicity. They also proved that the Kurucz new models, computed with the Castelli (1996) recipe of convection, are more realistic than previous ones because the computed Geneva indices are closer to the observed values.

In this paper, following the prescription of Castelli et al. (1997), we computed a new grid of models using the Lester (1998) version of the Kurucz ATLAS9 codes (Lester 1998). As a first attempt to investigate the effects of overshooting, we selected a few representative cases in parameter space. These cases are: temperatures at 4000,5000 and $6000 \mathrm{~K}$; surface gravity, $\log g=1.0$ and 4.0 ; metallicity, $[\mathrm{M} / \mathrm{H}]=[0.0]$, and $[-3.0]$. The selection of these models is motivated by their eventual utilization for studying stellar population in old systems and also to check for consistency of our results with those of Castelli et al. (1997).

In Fig. 1 we illustrate the temperature structure together with the variation of the fractional convective flux for the above models of solar metallicity with $\log g=4.0$. We can see from this figure that temperature structure differences of overshooting versus no-overshooting models are dominant in the case of $5000 \mathrm{~K}$ and $6000 \mathrm{~K}$ models. This is also reflected in the fractional flux due to convection. There is a consistency between our results and Castelli et al. (1997).

\subsection{Generating Synthetic Spectra}

The model fluxes provided by Kurucz are not at sufficient resolution to compute the Lick indices. We have used the above models and the Lester version of 
the Kurucz SYNTHE computer codes to synthesize spectra in the wavelength range 4800-5400 $\AA$. The spectral range includes seven Lick indices (see Table 1). In principle, a reliable line list should be used while computing the synthetic spectra. However, at present we are concerned with investigation of the role of "approximating overshooting" on the Lick indices. For this reason we used the line lists on the CDROM18, which includes atomic and molecular species, without implementing any modification on the line parameters. The details of the technique can be found in Gulati et al. (1991) and Chavez et al. (1997). A detailed description of the line data and procedure will be given elsewhere (Gulati \& Chavez 1998). As far as the other controlling parameters in the spectrum synthesis are concerned, computational resolution was set to 50000 in each synthetic spectrum. Each synthetic spectrum was degraded and rebinned to match the Lick/IDS system. As an example, in Fig. 2 we show the synthetic spectra in the central bandpasses of $\mathrm{H}_{\beta}$ and $\mathrm{Mg}_{b}$ for the model with parameters $\mathrm{T}_{\text {eff }}=5000 \mathrm{~K}, \log g=4.0$ and $[\mathrm{M} / \mathrm{H}]=0.0$.

\section{Preliminary Results}

Table 1. Lick/IDS indices in the wavelength range 4800-5400

\begin{tabular}{llcccc} 
Index & Units & Blue bandpass & Central bandpass & Red bandpass & Error \\
\hline $\mathrm{H}_{\beta}$ & $\AA$ & $4827.875-4847.875$ & $4847.875-4876.625$ & $4876.625-4891.625$ & 0.19 \\
$\mathrm{Fe} 5015$ & $\AA$ & $4946.500-4977.750$ & $4977.750-5054.000$ & $5054.000-5065.250$ & 0.41 \\
$\mathrm{Mg}_{1}$ & $\mathrm{mag}$ & $4895.125-4957.625$ & $5069.125-5134.125$ & $5301.125-5366.125$ & 0.006 \\
$\mathrm{Mg}_{2}$ & $\mathrm{mag}$ & $4895.125-4957.625$ & $5154.125-5196.625$ & $5301.125-5366.125$ & 0.007 \\
$\mathrm{Mg}_{b}$ & $\AA$ & $5142.625-5161.375$ & $5160.125-5192.625$ & $5191.375-5206.375$ & 0.20 \\
$\mathrm{Fe} 5270$ & $\AA$ & $5233.150-5248.150$ & $5245.650-5285.650$ & $5285.650-5318.150$ & 0.24 \\
$\mathrm{Fe} 5335$ & $\AA$ & $5304.625-5315.875$ & $5312.125-5352.125$ & $5353.375-5363.375$ & 0.22 \\
\hline
\end{tabular}

The Lick indices, as defined in Table 1 , were computed from the spectra using the REDUCEME package developed by Cardiel \& Gorgas (1997). The behavior of the indices with temperature indicates that in general all the indices increase with decreasing temperature, barring $\mathrm{H}_{\beta}$ which shows an opposite trend. As far as the dependence of the indices on gravity is concerned, we noticed that $\mathrm{Mg}_{b}$ is sensitive to gravity at the temperatures $5000 \mathrm{~K}$ and $6000 \mathrm{~K}$.

To evaluate the effect of overshooting and no-overshooting models on the Lick indices, we calculated the residuals defined as the difference between indices based on overshoot and non-overshoot models. Of all the indices in Table $1, \mathrm{H}_{\beta}$ and $\mathrm{Mg}_{b}$ have residuals values larger than the observational uncertainties given in column 6 taken from Trager et al. (1998). This suggests that among other sources of uncertainties, convection will be important to consider while building 
a grid of indices for stellar populations work. However, it needs to be investigated further by comparing empirical spectral libraries, especially with high signal to noise, with synthetic ones because as seen in Fig. 2 the core of the strong lines is affected.

Acknowledgements. The authors are pleased to thank M.L. Malagnini and C. Morossi for the collaboration and guidance during the early stages of the project and also acknowledge Bob Kurucz for providing the CDROMs. R.K. Gulati wishes to thank John Lester for fruitful discussion on adapting his version of the Kurucz codes to this project.

\section{References}

Barbuy, B., Erdelyi-Mendes, M., Milone, A. 1992, A\&AS, 93, 235

Cardiel, N., Gorgas, J. 1997, in preparation

Castelli, F. 1996, in: Model Atmospheres and Spectrum Synthesis, (eds.) S.J. Adelman, F. Kupka \& W.W. Weiss, ASP Conf. Proc. 108, p. 62

Castelli, F., Gratton, R.G., Kurucz, R.L. 1997, A\&A, 318, 841

Chavez, M., Malagnini, M.L., Morossi, C. 1995, ApJ, 440, 210

Chavez, M., Malagnini, M.L., Morossi, C. 1996, ApJ, 471, 726

Chavez, M., Malagnini, M.L., Morossi, C. 1997, A\&AS, 126, 267

Faber, S.M. 1973, ApJ, 179, 731

Gulati, R.K., Malagnini, M.L., Morossi, C. 1991, A\&A, 247, 447

Gulati, R.K., Malagnini, M.L., Morossi, C. 1993, ApJ, 413, 166

Gulati, R.K., Chavez, M. 1998, in preparation

Gustaffson, B., Bell, R.A., Eriksson, K., Nordlund, A. 1975, A\&A, 42, 407

Kunzli, M., North, P., Kurucz, R.L., Nicolet, B. 1997, A\&AS, 122, 51

Kurucz, R.L. 1979, ApJS, 40, 1

Kurucz, R.L. 1992, in: The Stellar Population of Galaxies, IAU Symp. 149, (eds.) B. Barbuy \& A. Renzini, Kluwer, Dordrecht, p. 225

Kurucz, R.L. 1993, CD-ROM 13, CD-ROM 18, and CD-ROM 19, Cambridge: Smithsonian Astrophys. Obs.

Lester, J.B. 1998, private communication

Mould, J. 1978, ApJ, 220, 434

Trager, S.C., Worthey, G., Faber, S.M., Burstein, D., Gonzalez, J.J. 1998, ApJS, 116,1

Tripicco, M.J., Bell, R.A. 1995, AJ, 110, 3035

Worthey, G. 1992, PhD thesis, University of California, Santa Cruz

Worthey, G. 1994, ApJS, 95, 107 


\section{Discussion}

Irwin: How much information do you lose from only using indices rather than the whole spectrum?

Gulati: Certainly, we get more information from the whole spectrum compared to spectral indices.

Hesser: You have worked extensively with these indices and the models. Can you give us, from your experience and intuition, some feeling regarding how much things will change as a result of including the overshooting effects?

Gulati: In my opinion, things will change in the simulation of spectra or spectral indices, as it has changed with regard to reproducing observational photometric properties with new convection models. 